\title{
Cell block technique as an additional tool in the diagnosis of ameloblastoma
}

\section{Maria Fernanda da Silva BELATTO(a) \\ Grasieli de Oliveira RAMOS(b) \\ Manoela Domingues MARTINS(c) \\ Liliane Janete GRANDO(d) \\ Elena Riet Correa RIVERO(d)}

(a) Postgraduate Program in Dentistry, Universidade Federal de Santa Catarina UFSC, Florianópolis, SC, Brazil.

(b) Postgraduate Program in Dentistry, Universidade Federal do Rio Grande do Sul - UFRGS, Porto Alegre, RS, Brazil.

(c) Department of Oral Pathology, School of Dentistry, Universidade Federal do Rio Grande do Sul - UFRGS, Porto Alegre, RS, Brazil.

(d) Department of Pathology, Universidade Federal de Santa Catarina - UFSC, Florianópolis, SC, Brazil.

Declaration of Interests: The authors certify that they have no commercial or associative interest that represents a conflict of interest in connection with the manuscript.

Corresponding Author:

Elena Riet Correa Rivero

E-mail: riet.elena@gmail.com

DOI: 10.1590/1807-3107BOR-2014.vol28.0044 Epub XXXXX, 2014

Submitted: Oct 22, 2013

Accepted for publication: Jun 02, 2014

Last revision: Aug 08, 2014
Abstract: The objective of this study was to evaluate the cytological content of ameloblastomas of the jaw. Nine cases of ameloblastoma were punctured, and the intralesional material was processed using the cell block technique. After centrifugation, the pellet obtained from the punctured material was fixed in formaldehyde and routinely processed to inclusion in paraffin. The obtained sections were stained with haematoxylin and eosin (H\&E). Immunohistochemical reactions against anti-pan-cytokeratin (AE1/AE3) were performed to measure the presence of epithelial cells. Cytological analyses of the obtained slides revealed the presence of epithelial cells (as evidenced by AE1/ AE3 labelling) and acellular amorphous eosinophilic materials. These cytological findings, in light of clinical and imaging data, can be helpful in the presumptive diagnosis of this disease entity by eliminating other possible diagnoses. Nonetheless further studies are needed in order to determine the nature of the acellular amorphous eosinophilic material and to ascertain the immunoprofile of epithelial cells.

Keywords: Biopsy, Fine-Needle; Cell Biology; Odontogenic Tumors; Ameloblastoma

\section{Introduction}

Ameloblastomas are benign odontogenic tumours that, despite having slow growth, are locally invasive, which results in a high rate of recurrence after conservative treatment. ${ }^{1}$ Given their aggressive biological behaviour, these odontogenic neoplasms are of great significance in dental practice. ${ }^{2}$ An important aspect regarding ameloblastomas is that they present similar clinical and radiographic manifestations to several other jaw cysts and benign osseous tumours with different clinical behaviours. The final diagnoses of these lesions have been obtained using intraosseous biopsy and histopathological examination, which represents an invasive procedure that could be complex in some bone sites and in systemically compromised patients. Within this context arises the need for developing and studying new diagnostic methods that reduce patient discomfort and facilitate the process of determining the presumptive diagnosis that is required for planning the treatment of lesions in the oral cavity, especially bone pathologies.

The needle aspiration (NA) is a clinical manoeuvre that involves puncturing a lesion for microscopic analysis. This analysis can be performed after smearing the aspirated fluid directly onto a glass slide or using the 
cell block technique. The cell block technique includes the centrifugation of the material punctured from the lesion to ensure the best use of the aspirated cells; therefore, this technique decreases cell dispersion of traditional cytology smears. ${ }^{3}$ It has been widely accepted that this analysis method increases cellular yield and improves diagnostic accuracy. Importantly, the cell block technique allows for the completion of several sections that could be submitted for other histochemical or immunohistochemical analyses. ${ }^{4}$ Previous studies have indicated that cell block is a good technique for the preliminary diagnosis of jawbone lesions. The studies performed by Rivero $\mathrm{et} \mathrm{al} .^{5}$ and Oenning et al..$^{6}$ demonstrated that cell block is a good auxiliary method to diagnose maxillomandibular lesions, especially Keratocystic Odontogenic Tumour (KCOT) and cystic lesions.

Given the clinical significance of ameloblastomas and the advantages of NA and the associated cell block technique, the objective of this study is to characterise the cytological content of ameloblastomas and evaluate the effectiveness of this technique in the presumptive diagnosis of these lesions.

\section{Methodology}

This study was approved by the Ethics Committee on Human Research of the Universidade Federal de Santa Catarina - UFSC (approval number 145/08).

Sampling included patients with intrabony jaw lesions with a clinical recommendation of aspiration. Patients were cared for at the Stomatology Ambulatory at the University Hospital and at the Dental Clinic of the UFSC. All participants were volunteers who were informed about the study and who were asked to sign an informed consent agreement. From 135 lesions punctured and processed using the cellblock technique, 9 cases that were histopathologically diagnosed as ameloblastoma were selected.

\section{Clinical procedure}

All patients with bone lesions underwent clinical and imaging examinations. The hypothesis of ameloblastoma and other cystic or tumour lesions was suggested. The needle aspiration was performed before conducting the biopsy, after obtaining adequate local anaesthesia, as follows: an 18-gauge needle con- nected to a $20 \mathrm{~mL}$ syringe was introduced into the lesion through either a thin area of the cortical bone or the point of perforation, if present. The syringe containing the collected material was immediately stored in a container with ice and sent to the Oral Pathology Laboratory for processing using the cell block technique.

\section{Laboratory procedure}

The material was transferred from the syringe into a test tube and centrifuged at a speed of 2000 rpm for 20 minutes. The obtained cell pellet was transferred onto absorbent paper and fixed in a $10 \%$ formaldehyde solution for 24 hours. The material was sequentially processed as follows: dehydration, clearing, impregnation and embedment in paraffin. Three-micrometre thick sections were obtained for haematoxylin and eosin (H\&E) staining and for immunohistochemical (IHC) reactions against anti-pan-cytokeratin (clone AE1/AE3 - Dako Corporation, Carpinteria, USA). IHC was performed as follows: sections were deparaffinised, hydrated in alcohol and immersed in 2\% $\mathrm{H} 2 \mathrm{O} 2$ in methanol for 30 minutes for the inhibition of endogenous peroxidase activity. Antigen retrieval was performed in a steamer for 20 minutes (Dako Corporation, Carpinteria, USA). The primary antibody was incubated for 18 hours at $4{ }^{\circ} \mathrm{C}$ and detected using the EnVision System (Dako Corporation, Carpinteria, USA). Diaminobenzidine (Biocare, Concord, USA) was used as a chromogen and the sections were counterstained with Mayer haematoxylin.

The stained slides were examined using light microscopy (Axiostar Plus, Carl Zeiss, Oberkochen, Germany) to characterise the types of material collected in relation to the presence of epithelial cells, inflammatory cells, erythrocytes or other components. This analysis was performed by an examiner who was blinded to the study and then by an oral pathologist; the results were subsequently agreed upon by consensus.

All of the cases used in this study presented a definitive diagnosis of ameloblastoma based on histopathological analysis according to criteria established by the World Health Organization (WHO). ${ }^{2}$ 


\section{Results}

Table 1 compares the clinical diagnosis with the definitive histopathological diagnosis of the surveyed cases. In three cases (33\%), the clinical hypothesis of ameloblastoma was not considered. In two (22\%) cases, the diagnostic possibilities were ameloblastoma and an additional bone lesion. The definitive diagnosis consisted of six cases of unicystic ameloblastoma (UA) and three cases of solid ameloblastoma (SA).

Table 1. Clinical diagnosis versus histopathological diagnosis

\begin{tabular}{ccc}
\hline Sample & Clinical diagnosis & $\begin{array}{c}\text { Histopathological } \\
\text { diagnosis }\end{array}$ \\
\hline 1 & Unicystic Ameloblastoma & Unicystic \\
/ Cysts and Odontogenic & Tumours & Ameloblastoma \\
2 & Unicystic Ameloblastoma & Ameloblastoma \\
3 & Paradental Cyst / Unicystic & Unicystic \\
& Ameloblastoma & Ameloblastoma \\
4 & Unicystic Ameloblastoma & Unicystic \\
& Ameloblastoma \\
5 & Unicystic Ameloblastoma & Unicystic \\
6 & Keratocystic odontogenic & Ameloblastoma \\
tumour & Ameloblastoma \\
7 & Keratocystic odontogenic & Ameloblastoma \\
& tumour & Unicystic \\
8 & Inflammatory Residual Cyst & Ameloblastoma \\
& Aneloblastoma & Unicystic \\
9 & Ameloblastoma
\end{tabular}

The data collected from the cytological analysis (Table 2) demonstrate that a considerable quantity of erythrocytes were present in six (66\%) cases, most likely resulting from the surgical procedure. The quantity of inflammatory cells varied between the anal- ysed cases, with the presence of both mononuclear and polymorphonuclear leukocytes. In seven (77\%) cases, the presence of epithelial cells was identified, and in most cases, these cells presented as insulated with a rounded form or a polygonal form (Figure 1). In specific cases, the presence of epithelial tissue fragments was observed (Figure 2). The existence of acellular amorphous eosinophilic materials was detected in five (55\%) cases (Figure 3). All epithelial cells were observed by IHC analysis utilising the AE1/AE3 antibody (Figure 4).

\section{Discussion}

The need for an effective establishment of a reliable presumptive diagnosis and, subsequently, a definitive diagnosis of lesions affecting the jaws is essential for planning an appropriate treatment. This need leads to the study of alternative diagnostic methods because a surgical procedure that results in an incisional or excisional biopsy for subsequent histological analysis can be complex in certain maxillomandibular sites and can have systemic contraindications in certain cases, such as uncompensated diabetes and coagulation abnormalities (e.g., Von Willebrand disease and haemophilia).

Recently, we have demonstrated the advantages and applicability of using punction aspiration followed by the cell block technique for cystic lesions of the jaws. ${ }^{5,6,7}$ The cytological analysis performed using materials prepared by the cell block technique provides an easy recognition of cells because the cytomorphological features remain well preserved after processing. ${ }^{8}$ An additional advantage of this tech-

Table 2. Cytological analysis

\begin{tabular}{|c|c|c|c|c|}
\hline Sample & Erythrocytes & Inflammatory cells & Epithelial cells* & $\begin{array}{c}\text { Acellular amorphous eosinophilic } \\
\text { materials }\end{array}$ \\
\hline 1 & Intense & Discrete & Absent & Present \\
\hline 2 & Absent & Scarce & Present & Absent \\
\hline 3 & Intense & Intense & Absent & Absent \\
\hline 4 & Intense & Intense & Present & Present \\
\hline 5 & Intense & Moderate & Present & Present \\
\hline 6 & Absent & Intense & Present & Present \\
\hline 7 & Absent & Intense & Present & Present \\
\hline 8 & Intense & Moderate & Present & Absent \\
\hline 9 & Intense & Moderate & Present & Absent \\
\hline
\end{tabular}

*Assisted by use of immunohistochemistry 


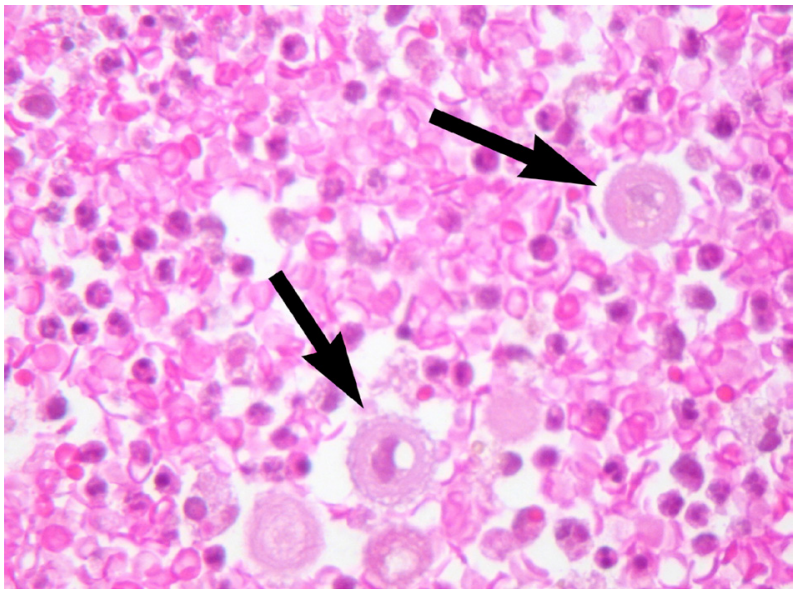

Figure 1. Presence of epithelial cells (arrows) (H\&E 400x).

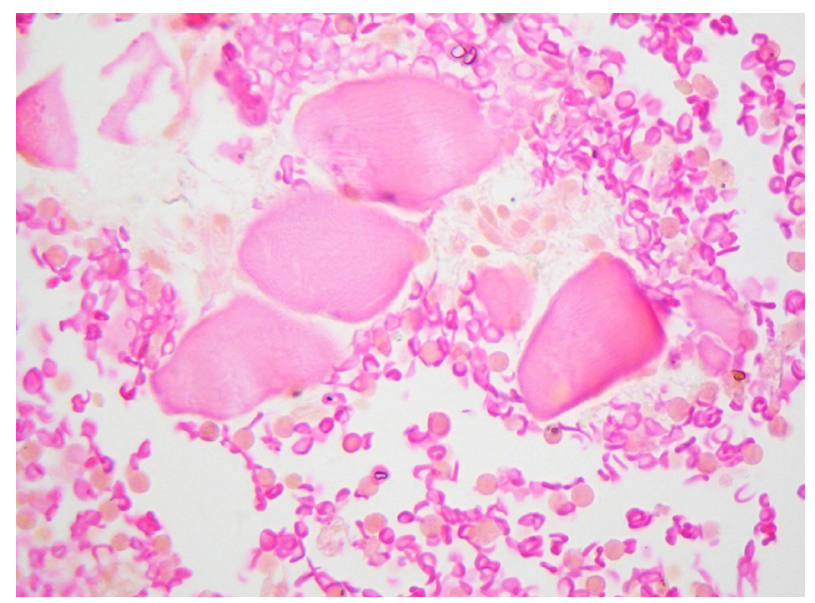

Figure 3. Presence of acellular amorphous eosinophilic material (H\&E 100x).

nique is the possibility of executing special stains, such as IHC, to identify structures. ${ }^{9}$ In the current study, it was possible to confirm the epithelial cells in the samples by IHC using the antibody AE1/AE3.

Our previous studies have shown that the evaluation of cytologic material obtained from puncturing the intraluminal content of cystic lesions of the jaws is especially useful in differentiating among the most frequent odontogenic cysts, such as radicular, residual and dentigerous cysts, as well as the KCOT, particularly when combined with a thorough clinical examination and imaging methods. ${ }^{5,6,7}$ According to Oenning et al., ${ }^{6}$ the identification of cholesterol crystal clefts in the cytology is useful in determin-

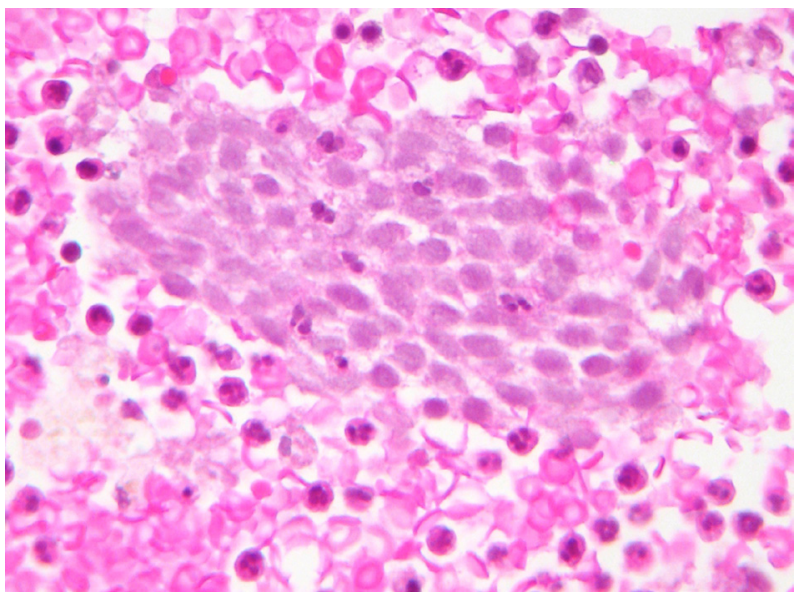

Figure 2. Fragment of epithelial tissue associated with inflammatory cells and erythrocytes (H\&E 400x).

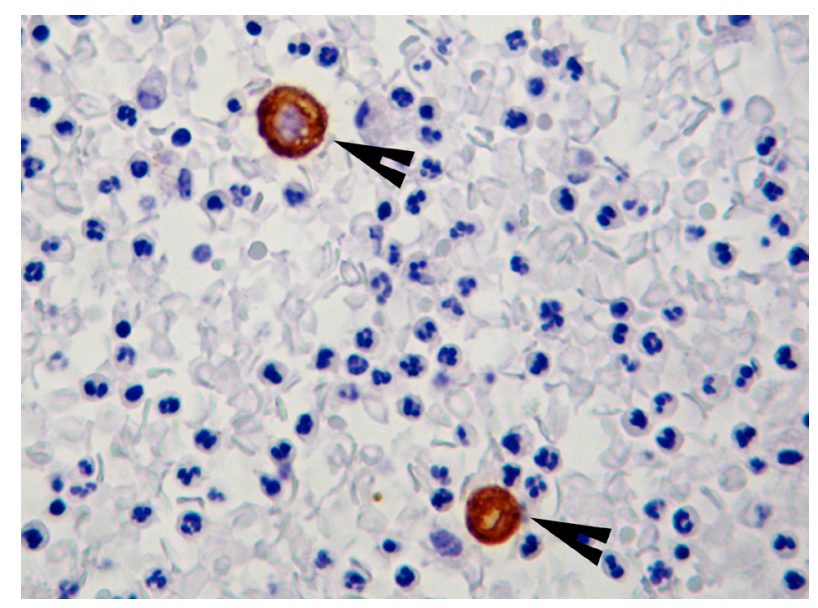

Figure 4. Immunohistochemical staining using the AE $1 / A E 3$ antibody, showing epithelial cells visualised as brown spots (arrowhead) (400x)

ing a presumptive diagnosis of odontogenic cysts of inflammatory aetiology, and identifying parakeratin is suggestive of KCOT, which in the case of KCOT, eliminates the need for incisional biopsy to perform the planning of therapy for these lesions. None of the cases of ameloblastoma in the present study indicated the presence of cholesterol crystal clefts or keratin in the cytological analyses.

Some studies have demonstrated the applicability of the cell block technique for determining the diagnosis of ameloblastomas of the jaws. ${ }^{10,11,12,13,14,15}$ In most cases, the presence of columnar peripheral cells arranged in palisading and inverted nuclear polarity is character- 
istic of ameloblastoma, enabling the determination of preoperative diagnosis. ${ }^{1011,12,13}$ However, note that most studies in which this methodology was performed in ameloblastomas used fine-needle aspiration cytology (FNAC) as a method of collecting material. In SA, it is easier to obtain material for evaluation by FNAC. In this study, approximately $70 \%$ of cases had a definitive diagnosis of UA; therefore, the material that was cytologically evaluated perhaps represented the intraluminal content of the lesions, justifying the predominant absence of specific structures in the sample studied.

In approximately $80 \%$ of the samples, we observed the presence of epithelial cells; however, an analysis of these cells, when isolated, generated many doubts due to the various forms that these cells can present after laboratory processing. Therefore, it was necessary to use the IHC technique to eliminate any doubts regarding the analysis of these cells. Notably, in most cases, the presence of epithelial cells was observed by using this technique alone.

In most cases, it was not possible to establish a diagnosis in isolated epithelial cells or in those that composed a piece of epithelial tissue because these cells showed no specific features or suggestions of ameloblastoma. However, it was possible to eliminate diagnostic hypotheses, such as KCOT and some cystic lesions, due to the absence of parakeratin and cholesterol crystal clefts, respectively.

Previous studies have shown that the expression of cytokeratin in cells obtained from aspiration is useful in the cytological diagnosis of KCOT. Vargas et al. ${ }^{16}$ showed the presence of epithelial cells in $100 \%$ of KCOT cases using the cell block technique with IHC for the confirmation of epithelial cells by AE1/ AE3 and CK 19 antibodies. However, according to our findings, cells positive for pan cytokeratin are present in ameloblastomas; therefore, the evaluation

\section{References}

1. Gardner DG, Pecak AMJ. The treatment of ameloblastoma based on pathologic and anatomic principles. Cancer. 1980 Dec;46(11):2514-9.

2. Barnes L, Everson JW, Reichart P, Sidransky D. World Health Organization classification of tumours. Pathology and genetics of head and neck tumours. Lyon: IARC Press; 2005; 430 p. of other structures, such as the presence of parakeratin, is important for the diagnosis of KCOT. 5,6,7

The presence of acellular amorphous eosinophilic materials, detected in 55\% of our samples, had not been observed in our previous studies with KCOT and cystic lesions, ${ }^{5,6,7}$ what makes us to conjecture about an epithelial origin. However, to completely understand the nature and origin of this material it would be needed a detailed IHC or electron microscopy study.

Although the cell block technique has many utilities and advantages, it is subject to limitations, such as small amounts of material for analysis or absence of material representative of the lesion, especially in cases of secondary infection. ${ }^{17}$ To minimise these limitations, it is suggested to use FNAC using a 24-gauge needle attached to a $10-\mathrm{ml}$ syringe and supported by a mechanical-syringe holder to facilitate aspiration as a method of collecting material for similar future studies.

\section{Conclusion}

The cytological analysis of the material punctured and processed using the cell block technique showed the presence of epithelial cells and acellular amorphous eosinophilic materials in most of the ameloblastoma cases studied. These cytological findings, associated with the absence of cholesterol crystal clefts or keratin, and the clinical and imaging data support the presumptive diagnosis of ameloblastoma by eliminating other possibilities, such as inflammatory cysts or KCOT. However, further studies are needed, especially in order to examine the immunoprofile of epithelial cells from cytological analysis of ameloblastomas, KCOT and odontogenic cysts, as well as to determine the exact nature of the acellular amorphous eosinophilic material presented in ameloblastomas.

3. Dereci O, Oztürk A, Günham O. The efficacy of fine needle aspiration cytology in the preoperative evaluation of parakeratotic odontogenic keratocysts. Acta Cytol. 2011 Mar-Apr;55(2):131-4.

4. Khan S, Omar T, Michelow P. Effectiveness of the cell block technique in diagnostic cytopathology. J Cytol. 2012 Jul;29(3):177-82. 
5. Rivero ER, Grando LJ, Menegat F, Claus JD, Xavier FM. Cell block technique as a complementary method in the clinical diagnosis for cyst-like lesions of the jaw. J Appl Oral Sci. 2011 May-Jun;19(3):269-73.

6. Oenning ACC, Rivero ER, Calvo MC, Meurer MI, Grando LJ. Evaluation of the cell block technique as an auxiliary method of diagnosing jawbone lesions. Braz Oral Res. 2012 Jul-Aug;26(4):355-9.

7. Rivero ER, Grando LJ, Ramos GO, Belatto MFS, Daniel FI. Utility of cell block in the cytological preoperative diagnosis of keratocystic odontogenic tumor. Pathol Res Pract. 2014 Apr;210(4):224-7.

8. Nathan NA, Narayan E, Smith MM, Horn MJ. Cell block cytology. Improved preparation and its efficacy in diagnostic cytology. Am J Clin Pathol. 2000 Oct;114(4):599-606.

9. Saleh HA, Hammoud J, Zakaria R, Khan AZ. Comparison of thin-prep and cell block preparation for the evaluation of thyroid epithelial lesions on fine needle aspiration biopsy. Cytojournal. 2008 Mar 25;5(3):1-7.

10. Günhan O, Finci R, Celasun B, Demiriz M. A case of ameloblastoma diagnosed by fine-needle aspiration cytology. J Nihon Univ Sch Dent. 1989 Dec;31(4):565-9.
11. Mathew S, Rappaport K, Ali SZ, Busseniers AE, Rosenthal DL. Ameloblastoma: cytologic findings and literature review. Acta Cytol. 1997 Jul-Aug;41(4):955-60.

12. Radhika S, Nijhawan R, Das A, Dey P. Ameloblastoma of the mandible: diagnosis by fine-needle aspiration cytology. Diagn Cytopathol. 1993 May;9(3):310-3.

13. Stamatakos MD, Houston GD, Fowler CB, Boyd E, Solanki $\mathrm{PH}$. Diagnosis of ameloblastoma of the maxilla by fine needle aspiration: a case report. Acta Cytol. 1995 Jul-Aug;39(4):817-20.

14. Uçok O, Doğan N, Uçok C, Günhan O. Role of fine needle aspiration cytology in the preoperative presumptive diagnosis of ameloblastoma. Acta Cytol. 2005 Jan-Feb;49(1):38-42.

15. Bisht S, Kotwal SA, Gupta P, Dawar R. Role of fine needle apiration cytology in preoperative diagnosis of ameloblastoma. Indian J Cancer. 2009 Oct-Dec;46(4):348-50.

16. Vargas PA, Cruz Perez DE, Mata GM, Almeida OP, Jones AV, Gerhard R. Fine needle aspiration cytology as an additional tool in the diagnosis of odontogenic keratocyst. Cytopathol. 2007 Dec;18(6):361-6.

17. August M, Faquin WC, Ferraro NF, Kaban LB. Fine-needle aspiration biopsy of intraosseous jaw lesions. J Oral Maxillofac Surg. 1999 Nov;57(11):1282-6 